\title{
Short-term diagnostic stability among re-admitted psychiatric in-patients in Eldoret, Kenya
}

\author{
L Atwoli', D Ndambuki ${ }^{2}$, P Owiti ${ }^{2}$, G Manguro ${ }^{2}$ N Omulimi ${ }^{2}$ \\ 1Department of Mental Health, Moi University School of Medicine, Eldoret, Kenya \\ 2Ministry of Medical Services, Government of Kenya, Nairobi, Kenya
}

\begin{abstract}
Objective: To determine the prospective and retrospective consistency of diagnoses among readmitted psychiatric in-patients at the Moi Teaching and Referral Hospital in Eldoret, Kenya. Method: Admission and discharge diagnoses among a consecutive sample of 114 psychiatric in-patients readmitted at the Moi Teaching and Referral Hospital between August and December 2009 were compared. Results: The commonest diagnoses at admission were schizophrenia spectrum disorders (47.4\%) and bipolar spectrum disorders (30.7\%). Overall diagnostic stability as measured by prospective consistency in this study was 72.8\%.The most stable diagnostic category was Major Depressive Disorder (100\% prospective and retrospective consistency), followed by Bipolar Disorders (91.4\% prospective consistency, 69.6\% retrospective consistency) and Substance-related disorders (87.5\% prospective consistency, 50\% retrospective consistency). Schizophrenia-spectrum disorders had a prospective consistency of $75.9 \%$ and a retrospective consistency of $87.2 \%$. 'Other Psychotic Disorders' (acute psychotic episode and psychotic disorder not otherwise specified) had the lowest diagnostic stability, with both prospective and retrospective consistency being $0 \%$. Conclusion: Mood disorders and substance-related disorders have the highest diagnostic stability among readmitted psychiatric inpatients in a low-income country.
\end{abstract}

Key Words: Diagnostic Stability; Psychiatric Inpatients; Low and Middle income countries

Received: $25-02-2011$

Accepted: 09-05-201 1

doi: http://dx.doi.org/10.4314/ajpsy.v15i2.15

\section{Introduction}

Diagnostic stability, or the degree to which a diagnosis remains constant at consecutive patient assessments, is a useful index for measuring the reliability of various components of a patient assessment in various settings. ${ }^{1}$ Diagnostic changes over time may reflect the natural evolution of an illness, the emergence of previously unavailable information or unreliability of measurements. ${ }^{2}$ However, poor diagnostic stability has potential implications not only in terms of the patient's health but also in terms of service planning and resource allocation. ${ }^{1}$ The significance of frequent changes in diagnosis and management plans cannot be overstated especially in resource-limited settings such as Sub-Saharan Africa.

Many prospective studies have concluded that schizophrenia is the most stable diagnosis (up to 90\%) with schizophreniform disorder, schizo-affective disorder and psychotic disorder not otherwise specified amongst the least stable initial diagnoses. ${ }^{1-10}$ Stability has been reported to be greatest for in-patients as compared to out-patient diagnoses. ${ }^{2}$ A possible explanation for this is that it may be easier to diagnose a condition when symptom severity is at its highest, as in emergency or inpatient settings, than it would be among outpatients. Veen et al reported that schizophrenic disorders are likely to be under-diagnosed at first presentation compared to other diagnoses. ${ }^{11}$

Most of the studies on diagnostic stability have been carried out in Western settings, and there is very little literature from Africa and other low-income regions on this topic. This study therefore set out to determine the diagnostic stability of various psychiatric diagnoses among re-admitted patients using prospective and retrospective consistency as measures of stability. The information generated from this study will hopefully be useful in filling the knowledge gap and help in improving patient management in resource-poor settings.

\section{Method}

Setting

This study was conducted between the months of August and December 2009 at the Moi Teaching and Referral Hospital 
(MTRH) in Eldoret town, Kenya. Eldoret town is situated about 320 kilometres northwest of Kenya's capital city, Nairobi, and the MTRH is the second largest public tertiary level hospital in Kenya. It serves a population of over 20 million people from Western Kenya and neighbouring countries, including Uganda, Southern Sudan, Tanzania and even Ethiopia.

MTRH has a small inpatient psychiatric unit with a capacity for 22 beds. However, the unit is often overcrowded, at times accommodating upto 80 inpatients at a time. Admission assessments may be carried out by a qualified psychiatrist or, more often an emergency room medical officer with a general medical practice licence. Discharge diagnoses are assigned by a team led by a psychiatrist and consisting of a clinical psychologist, nurses, social workers and occupational therapists.

Statistics from the unit showed that the average turnaround time (duration between admission and discharge) in the unit was 35 days at the time of the study.

\section{Study Design}

This study was a cross-sectional descriptive study using consecutive sampling over a period of five months to select psychiatric in-patients with at least one previous admission. Data was collected as part of a larger study examining the factors associated with readmission at the MTRH, and all participants were required to have had at least one previous admission with similar symptoms.

\section{Participants}

A total of 114 inpatients were interviewed for the study once the decision to discharge them from the ward had been made by the treating team. This was done in order to ensure they were capable of giving consent to participate in the study.

\section{Procedure}

Ethical review and approval was granted by the Institutional Research and Ethics Committee (IREC) of Moi University and MTRH, and only adult in-patients were selected for the study. All participants gave informed consent to participate in the study to have their hospital records used for the study. Data was then collected from the patients' records, including sociodemographic data, previous admissions, diagnoses made and the treatment that was given.

\section{Data storage and analysis}

The collected data was entered into a Microsoft Excel database and analysed using the Statistical Package for Social Sciences (SPSS ver. 12.0).

Two measures of diagnostic stability were used for each diagnostic category.

The first measure, prospective consistency, corresponded to the percentage of patients who retained the admission diagnosis at the time of discharge; this value would equal the positive predictive value, assuming that the discharge diagnosis was the gold standard.

The second measure, retrospective consistency, corresponded to the proportion of patients with a particular diagnosis on discharge who had received the same diagnosis at admission; this value would represent the sensitivity of the initial diagnostic process if the discharge diagnosis is considered to be the gold standard.

\section{Results}

Socio-demographic characteristics

A total of 114 patients were recruited for the study. As illustrated in Table I, $64 \%$ of the participants were males. In this sample, only 35\% were married, and the main source of income for the majority of the participants was farming (58.8\%).

The mean age of the participants was 34.7 years (18-71, s.d. 10.8) while the mean number of years of schooling was 8.9 (0-18, s.d. 3.6). The mean number of admissions for the same indication was 4.73 (2-25, s.d. 3.38). All the diagnoses at admission and at discharge were combined into 5 major DSM IV-TR ${ }^{12}$ Axis I diagnostic groups and one group with other diagnoses, as illustrated in Table II.

\begin{tabular}{|l|l|}
\hline \multicolumn{2}{|l|}{ Table I: Socio-demographic characteristics } \\
\hline VARIABLE (N=114) & FREQUENCY (\%) \\
\hline GENDER & $73(64)$ \\
Male & $41(36)$ \\
\hline Female & \\
\hline MARITAL STATUS & $62(54.4)$ \\
Single & $40(35.0)$ \\
Married & $6(5.3)$ \\
Divorced/separated & $6(5.3)$ \\
Widowed & \\
\hline SOURCE OF INCOME & $67(58.8)$ \\
Farming & $20(17.5)$ \\
Salaried Employment & $17(14.9)$ \\
Self-employment & $10(8.8)$ \\
Other & \\
\hline
\end{tabular}

\begin{tabular}{|c|c|c|}
\hline Diagnoses $(N=114)$ & $\begin{array}{l}\text { Frequency at } \\
\text { admission (\%) }\end{array}$ & $\begin{array}{l}\text { Frequency at } \\
\text { discharge (\%) }\end{array}$ \\
\hline $\begin{array}{l}\text { Schizophrenia Spectrum } \\
\text { Schizophrenia } \\
\text { Schizoaffective }\end{array}$ & $\begin{array}{l}54(47.4) \\
52(45.7) \\
2(1.7)\end{array}$ & $\begin{array}{l}47(41.2) \\
47(41.2) \\
0\end{array}$ \\
\hline $\begin{array}{l}\text { Bipolar Spectrum } \\
\text { Bipolar I disorder } \\
\text { Bipolar II disorder }\end{array}$ & $\begin{array}{l}35(30.7) \\
34(29.8) \\
1(0.9)\end{array}$ & $\begin{array}{l}46(40.4) \\
46(40.4) \\
0\end{array}$ \\
\hline Major Depressive Disorder & $3(2.6)$ & $3(2.6)$ \\
\hline $\begin{array}{l}\text { Substance related Disorders } \\
\text { Alcohol abuse } \\
\text { Alcohol dependence } \\
\text { Alcohol induced mood disorder } \\
\text { Other Substance abuse } \\
\text { Substance induced psychosis }\end{array}$ & $\begin{array}{l}8(7.0) \\
1(0.9) \\
4(3.5) \\
0 \\
2(1.7) \\
1(0.9)\end{array}$ & $\begin{array}{l}14(12.3) \\
3(2.6) \\
4(3.5) \\
2(1.8) \\
2(1.8) \\
3(2.6)\end{array}$ \\
\hline $\begin{array}{l}\text { Other Psychotic disorders } \\
\text { Acute Psychotic Episode } \\
\text { Non-specified psychosis }\end{array}$ & $\begin{array}{l}14(12.3) \\
1(0.9) \\
13(11.4)\end{array}$ & $\begin{array}{l}1(0.9) \\
1(0.9) \\
0\end{array}$ \\
\hline $\begin{array}{l}\text { Other diagnoses } \\
\text { Temporal Lobe Epilepsy } \\
\text { Mental Retardation } \\
\text { No Diagnosis }\end{array}$ & $\begin{array}{l}0 \\
0 \\
0 \\
0\end{array}$ & $\begin{array}{ll}3 & (2.6) \\
1 & (0.9) \\
1 & (0.9) \\
1 & (0.9)\end{array}$ \\
\hline
\end{tabular}


The majority of the patients on admission were diagnosed with schizophrenia (47.4\%) or bipolar spectrum disorders (30.7\%).

\section{Overall Diagnostic stability}

Out of the 114 patients, 83 retained their admission diagnosis at the time of discharge, giving an overall prospective consistency of $72.8 \%$. Table III shows the prospective and retrospective consistency of the various diagnoses, as well as the data from which those figures are derived.

\section{Schizophrenia}

A total of 54 patients (47.4\%) had a diagnosis of a schizophrenia spectrum disorder at admission. On discharge, the frequency of this diagnostic category had reduced to 47 (41.2\%). As shown in Table III, the prospective consistency for the schizophrenia spectrum disorders was 75.9\% while the retrospective consistency was $87.2 \%$. Thirteen patients had their diagnosis change from schizophrenia spectrum to another diagnosis, and $69.2 \%$ of these were diagnosed with a bipolar spectrum disorder, while others were diagnosed with Substance related disorders (2 patients) and other psychotic disorder (1 patient). One patient was found not to have any Axis I diagnosis.

\section{Bipolar spectrum disorders}

Among the respondents, 35 (30.7\%) were diagnosed with a bipolar spectrum disorder on admission, while on discharge 46 patients (40.4\%) had this diagnosis. The prospective consistency for this diagnosis was $91.4 \%$, while the retrospective consistency was 69.6\%. Among the 3 patients whose diagnosis was changed by the time of discharge, 2 were diagnosed with a schizophrenia spectrum disorder while one was diagnosed with a substance-related disorder.

\section{Major depressive disorder}

Only 3 patients (2.6\%) were diagnosed with major depressive disorder at both admission and discharge. Both the prospective consistency and the retrospective consistency for this disorder were 100\%.
Substance-related disorders

Eight respondents (7.0\%) were diagnosed with a substancerelated disorder on admission, while on discharge 14 (12.3\%) respondents had this diagnosis. The prospective consistency of a diagnosis of substance-related disorder was $87.5 \%$, while the retrospective consistency was 50\%. Out of the 8 patients admitted with this diagnosis, only one had the diagnosis changed to bipolar spectrum disorders.

Other psychotic disorders

At admission, 14 patients (12.3\%) were diagnosed with 'Other Psychotic Disorders' (Acute psychotic episode and psychosis not otherwise specified) while on discharge only one patient $(0.9 \%)$ had this diagnosis. All patients admitted with a diagnosis in this category received different diagnoses on discharge. Both the prospective and the retrospective consistency for this diagnostic category were $0 \%$.

\section{Discussion}

The usefulness of assessing short-term diagnostic stability has not been established before, in addition there are very few studies assessing this construct from developing countries. Studies elsewhere have mostly assessed longterm diagnostic stability over months or years ${ }^{2,4,6,8,9,13}$, and a majority have concentrated on a single diagnostic category such as schizophrenia, major depressive disorder or bipolar disorder. ${ }^{1,3,5,11,14-26}$

This present study set out to assess diagnostic stability in a resource-poor setting, and the findings indicate that the rate of short-term diagnostic stability is surprisingly low (72.8\%) despite the fact that the assessments were often carried out within a few days of each other. The average time between admission and discharge at the MTRH was 35 days, meaning that the admission and discharge assessments were carried out just over one month apart on average. Other studies with longer follow-up periods have demonstrated similar findings as far as prospective consistency is concerned. ${ }^{1,11,26}$

The rapid shift in diagnosis in this study may be explained not so much by evolution of the illness as suggested by Schwartz et $\mathrm{al}^{26}$, but by the possibility of

\section{Table III: Diagnostic stability indices}

\begin{tabular}{|c|c|c|c|c|c|c|c|c|c|}
\hline \multirow[b]{2}{*}{ Diagnosis at admission } & \multicolumn{7}{|c|}{ Diagnosis on discharge } & \multirow[b]{2}{*}{$\begin{array}{l}\text { \% Prospective } \\
\text { consistency }\end{array}$} & \multirow[b]{2}{*}{$\begin{array}{l}\text { \% Retrospective } \\
\text { consistency }\end{array}$} \\
\hline & None & SSD & $B S D$ & $M D D$ & SRD & $O P D$ & Others & & \\
\hline $\operatorname{SSD}(n=54)$ & 0 & 41 & 9 & 0 & 2 & 1 & 1 & 75.9 & 87.2 \\
\hline $\operatorname{BSD}(n=35)$ & 0 & 2 & 32 & 0 & 1 & 0 & 0 & 91.4 & 69.6 \\
\hline $\operatorname{MDD}(n=3)$ & 0 & 0 & 0 & 3 & 0 & 0 & 0 & 100 & 100 \\
\hline $\operatorname{SRD}(n=8)$ & 0 & 0 & 1 & 0 & 7 & 0 & 0 & 87.5 & 50 \\
\hline OPD $(n=14)$ & 1 & 4 & 4 & 0 & 4 & 0 & 1 & 0 & 0 \\
\hline Total $(\mathrm{N}=114)$ & 1 & 47 & 46 & 3 & 14 & 1 & 2 & 72.8 & \\
\hline
\end{tabular}


diagnostic errors at admission. Admission diagnoses in this setting are often made by medical officers in the emergency room, many of whom have limited exposure to psychiatric training and work in high-pressure environments in which diagnostic errors are highly likely. Once a patient is admitted, however, more systematic assessments are made by a team comprising of psychiatrists, a clinical psychologist, nurses, social workers and occupational therapists. The larger number of specialists involved in the diagnostic process, the amount of time spent assessing the patients and the more consistent use of collateral sources of information may serve to improve the likelihood of a correct diagnosis being made.

To improve the diagnostic accuracy in this facility, it is recommended that decision-making algorithms and standard operating procedures be developed and implemented at the point of initial assessment of psychiatric patients. Further, it may be necessary to insist that the clinicians responsible for reviewing patients in emergency settings make better use of available patient records, especially in instances where there is little reliable corroborative information.

In this study, the prospective consistency of schizophrenia spectrum disorders (75.9\%) was relatively lower than that found in other studies, most of which reported rates averaging 90\%, 1,10,11,17,26,27 This is surprising due to the fact that all the patients in this study had at least one previous admission with similar symptoms, and their records were often available to the admitting medical officers. The chronic deteriorating course of schizophrenia and related disorders would suggest a high likelihood of greater diagnostic stability, but this was not the case in this study. Lack of attention to detail probably due to the highpressure environment of the emergency room may partly explain this, as may poor record-keeping and retrieval systems at the hospital.

Retrospective consistency of schizophrenia spectrum disorders was, however, relatively high, suggesting that overdiagnosis was more likely to occur in this diagnostic category than misdiagnosis. The tendency by many nonpsychiatrist physicians and medical officers to label most patients with abnormal behaviour as schizophrenic may explain this phenomenon. Chen et al. ${ }^{5}$ described a similar finding in a large longitudinal study at an urban acute care psychiatric hospital in the US. However, in other settings, a tendency to under-diagnose schizophrenia has been reported. ${ }^{11,26}$ Over-diagnosis of schizophrenia in this study increases the risk of stigma associated with a chronic mental illness and raises the possibility of poorer outcomes for patients and their families as reported elsewhere.

Compared to schizophrenia, the diagnostic stability for mood disorders including bipolar spectrum disorders and major depressive disorder was high. This contrasts with other studies that have consistently found higher rates for schizophrenia than any of the mood disorders. ${ }^{1,10,11,14,21,25-28}$

Due to the very small number of patients admitted with a diagnosis of major depressive disorder, it is difficult to make any conclusions regarding the prospective and retrospective consistency of this diagnostic category. However, in this setting, patients with depression are rarely admitted in hospital, and only those with severe symptomatology are brought to hospital for treatment. Such symptoms include suicidal ideation and attempts as well as severe vegetative symptoms like sleep disturbance, poor feeding and weight changes.

Bipolar disorders in this study had a higher prospective consistency than that found in several other studies. ${ }^{10,11,26}$ The dramatic presentation of manic symptoms may have made it easier to make a diagnosis in these patients.

A large number of patients initially diagnosed with schizophrenia were later diagnosed as having bipolar disorder, giving rise to the relatively low retrospective consistency (69.6\%). This shows that although an admission diagnosis of bipolar disorder in this setting was often quite accurate, over 30\% of patients with bipolar disorder were misdiagnosed at admission, with most of them getting a diagnosis of schizophrenia or other psychotic disorders.

Similarly, substance-related disorders had a relatively high prospective consistency (87.5\%) and poor retrospective consistency (50\%). This is surprising given evidence suggesting that even in the general population in low-income countries substance use is often assumed to be a major cause of mental illness. ${ }^{29}$ Many patients initially diagnosed with schizophrenia and other psychotic disorders were later found to have substance-related disorders, supporting the conclusion that over-diagnosis of psychotic disorders is common. Despite the low numbers of patients diagnosed with substance-related disorders, it may be necessary to encourage a higher degree of vigilance in order to correctly diagnose and manage substance-related disorders in this population.

The least stable diagnostic category in this study was 'Other Psychotic Disorders', a category comprising of diagnoses such as acute psychotic episode and psychotic disorder not otherwise specified. This is consistent with findings in other studies, though none of the studies reviewed returned both prospective and prospective consistencies of $0 \% .{ }^{11,26,27}$ Most of those admitted with this diagnosis were later diagnosed to have schizophrenia spectrum disorders, bipolar disorders and substance-related disorders as shown in Table III. Interestingly, one respondent admitted with this diagnosis was later found to have no diagnosis on any of the axes, while another had no Axis I diagnosis.

The poor stability of the 'Other Psychotic Disorder' category may be attributed to its nature as a provisional diagnosis in most cases in this setting. After evaluation in the ward the patients were given new diagnoses based on their symptomatology. Indeed the DSM IV TR ${ }^{12}$ allows a diagnosis of Psychotic Disorder Not Otherwise Specified to be made in cases with psychotic symptomatology about which there is inadequate or contradictory information, increasing the likelihood that the diagnosis would change as soon as new corroborative information is made available.

\section{Limitations}

The main limitations of this study are the relatively short duration of follow-up of the subjects and the small sample size. The short duration of follow-up was necessitated by limited funding for this study, as well as poor record-keeping that entailed use of physical records stored in several locations within the hospital. An electronic medical records system will in future make it easier to conduct larger studies 
over longer periods of time than the present study. The small sample size precludes use of this data to generalise to situations elsewhere, even where the socio-economic circumstances are similar. However, it is hoped that these findings will be useful in generating hypotheses that may be used in designing larger longitudinal studies on diagnostic stability in a resource-poor setting.

An important strength of this study is that the findings are based on routine diagnostic procedures carried out during regular clinical practice, and therefore are likely to provide a good snapshot of real-world practice at this facility. The potential to use the information to improve clinical decisionmaking is therefore great, for instance in the recommendations to make better use of available clinical records and to develop decision-making algorithms or standard operating procedures at the point of first assessment.

\section{Conclusion}

This study of readmitted psychiatric inpatients in a lowincome setting has demonstrated that mood disorders and substance-related disorders had the highest diagnostic stability based on prospective consistency. Studies involving larger sample sizes and longer follow-up periods are recommended to confirm these findings and establish some of the factors behind poor short-term diagnostic stability in this setting

\section{Acknowledgements}

The authors would like to acknowledge Mr Julius Atitwa, Nursing Officer in the MTRH psychiatric unit for assisting with data collection.

\section{References}

1. Whitty P, Clarke M, MCTigue O, Browne S, Kamali M, Larkin C, et al. Diagnostic stability four years after a first episode of psychosis. Psychiatr Serv 2005;56:1084-8.

2. Baca-Garcia E, Perez-Rodriguez MM, Basurte-Villamor I, Fernandez del Moral AL, Jimenez-Arriero MA, Gonzalez de Rivera JL, et al. Diagnostic stability of psychiatric disorders in clinical practice. Br J Psychiatry 2007;190:210-6.

3. Amara IB. Consistency in the diagnosis of the functional psychoses. Can Psychiatr Assoc J 1978;23:329-36.

4. Babigian HM, Gardner EA, Miles HC, Romano J. Diagnostic Consistency and Change in a Follow-up Study of 1215 Patients. Am J Psychiatry 1965;121:895-901.

5. Chen YR, Swann AC, Burt DB. Stability of diagnosis in schizophrenia. Am J Psychiatry 1996;153:682-6.

6. Kendell RE. The stability of psychiatric diagnosis. Br J Psychiatry 1974;124:352-6.

7. Marneros A, Deister A, Rohde A. Stability of diagnoses in affective, schizoaffective and schizophrenic disorders. Cross-sectional versus longitudinal diagnosis. Eur Arch Psychiatry Clin Neurosci 1991;241:187-92.

8. Rabinowitz J, Slyuzberg M, Ritsner M, Mark M, Popper M, Ginath Y. Changes in diagnosis in a 9-year national longitudinal sample. Compr Psychiatry 1994;35:361-5.

9. Stanton MW, Joyce PR. Stability of psychiatric diagnoses in New Zealand psychiatric hospitals. Aust N Z J Psychiatry 1993;27:2-8.

10. Tsuang MT, Woolson RF, Winokur G, Crowe RR. Stability of psychiatric diagnosis. Schizophrenia and affective disorders followed up over a 30- to 40-year period. Arch Gen Psychiatry $1981 ; 38: 535-9$.

11. Veen ND, Selten JP, Schols D, Laan W, Hoek HW, van der Tweel I, et al. Diagnostic stability in a Dutch psychosis incidence cohort. Br J Psychiatry 2004;185:460-4

12. American Psychiatric Association. Diagnostic and Statistical Manual of Mental Disorders. 4th ed. Washington, DC: American Psychiatric Association, 2000.

13. Vollebergh WA, Iedema J, Bijl RV, de Graaf R, Smit F, Ormel J. The structure and stability of common mental disorders: the NEMESIS study. Arch Gen Psychiatry 2001;58:597-603.

14. Amin S, Singh SP, Brewin J, Jones PB, Medley I, Harrison G. Diagnostic stability of first-episode psychosis. Comparison of ICD10 and DSM-III-R systems. Br J Psychiatry 1999;1 75:537-43.

15. Baca-Garcia E, Perez-Rodriguez MM, Basurte-Villamor I, LopezCastroman J, Fernandez del Moral AL, Jimenez-Arriero MA, et al. Diagnostic stability and evolution of bipolar disorder in clinical practice: a prospective cohort study. Acta Psychiatr Scand 2007;1 15:473-80.

16. Bromet EJ, Dunn LO, Connell MM, Dew MA, Schulberg HC. Longterm reliability of diagnosing lifetime major depression in a community sample. Arch Gen Psychiatry 1986;43:435-40.

17. Bromet EJ, Naz B, Fochtmann LJ, Carlson GA, Tanenberg-Karant M. Long-term diagnostic stability and outcome in recent first-episode cohort studies of schizophrenia. Schizophr Bull 2005;31:639-49.

18. Chen YR, Swann AC, Johnson BA. Stability of diagnosis in bipolar disorder. J Nerv Ment Dis 1998;186:17-23.

19. Coryell W, Winokur G, Shea T, Maser JD, Endicott J, Akiskal HS. The long-term stability of depressive subtypes. Am J Psychiatry 1994;151:199-204.

20. Fennig S, Kovasznay B, Rich C, Ram R, Pato C, Miller A, et al. Sixmonth stability of psychiatric diagnoses in first-admission patients with psychosis. Am J Psychiatry 1994;151:1200-8.

21. Kessing LV. Diagnostic stability in bipolar disorder in clinical practise as according to ICD-10. J Affect Disord 2005;85:293-9.

22. Kessing LV. Diagnostic stability in depressive disorder as according to ICD-10 in clinical practice. Psychopathology 2005;38:32-7.

23. Lenz G, Simhandl C, Thau K, Berner P, Gabriel E. Temporal stability of diagnostic criteria for functional psychoses. Results from the Vienna follow-up study. Psychopathology 1991;24:328-35.

24. Mason P, Harrison G, Croudace T, Glazebrook C, Medley I. The predictive validity of a diagnosis of schizophrenia. A report from the International Study of Schizophrenia (ISoS) coordinated by the World Health Organization and the Department of Psychiatry, University of Nottingham. Br J Psychiatry 1997;170:321-7.

25. Schimmelmann BG, Conus P, Edwards J, McGorry PD, Lambert M. Diagnostic stability 18 months after treatment initiation for firstepisode psychosis. J Clin Psychiatry 2005;66:1239-46.

26. Schwartz JE, Fennig S, Tanenberg-Karant M, Carlson G, Craig T, Galambos $N$, et al. Congruence of diagnoses 2 years after a firstadmission diagnosis of psychosis. Arch Gen Psychiatry 2000;57:593-600.

27. Hollis C. Adult outcomes of child-and adolescent-onset schizophrenia: diagnostic stability and predictive validity. Am J Psychiatry 2000;157:1652-9.

28. Vieta E, Phillips ML. Deconstructing bipolar disorder: a critical review of its diagnostic validity and a proposal for DSM-V and ICD11. Schizophr Bull 2007;33:886-92.

29. Kabir M, Iliyasu Z, Abubakar IS, Aliyu MH. Perception and beliefs about mental illness among adults in Karfi village, northern Nigeria. BMC Int Health Hum Rights 2004;4:3. 